\title{
Flow modelling of quasi-Newtonian fluids in two-scale fibrous fabrics
}

\author{
Advanced simulations
}

\author{
Amine Ammar ${ }^{1,2}$. Emmanuelle Abisset-Chavanne ${ }^{3}$ - Francisco Chinesta ${ }^{3}$. \\ Roland Keunings 4
}

\begin{abstract}
Permeability is the fundamental macroscopic material property needed to quantify the flow in a fibrous medium viewed as a porous medium. Composite processing models require the permeability as input data to predict flow patterns and pressure fields. In a previous work, the expressions of macroscopic permeability were derived in a double-scale porosity medium for both Newtonian and generalized Newtonian (shear-thinning) resins. In the linear case, only a microscopic calculation on a representative volume is required, implying as many microscopic calculations as there are representative microscopic volumes in the whole fibrous structure. In the non-linear case, and even when the porous microstructure can be described by a unique representative volume, a large number of microscopic calculations must be carried out as the microscale resin viscosity depends on the macroscopic velocity, which in turn depends on the permeability that results from a microscopic calculation. An original and efficient offlineonline procedure was proposed for the solution of non-linear flow problems related to generalized Newtonian fluids in
\end{abstract}

porous media. In this paper, this procedure is generalized to quasi-Newtonian fluids in order to evaluate the effect of extensional viscosity on the resulting upscaled permeability. This work constitutes a natural step forward in the definition of equivalent saturated permeabilities for linear and non-linear fluids.

Keywords Permeability - Computational homogenization · Quasi-Newtonian fluids · Generalized Newtonian fluids · Extensional viscosity · Composite materials · Model order reduction · Proper generalized decomposition $\cdot$ Parametric solutions

\section{Introduction}

Isothermal flows of complex fluids in complex microstructures can be simulated by solving the momentum and mass balance equations and a suitable rheological constitutive model. For inertialess incompressible flows, these balance equations read,

$\nabla \cdot \boldsymbol{\sigma}=\mathbf{0}$

and

$\nabla \cdot \mathbf{v}=0$

respectively. Here, $\sigma$ is the Cauchy stress tensor and $\mathbf{v}$ the velocity field, both defined at time $t$ at each point within the fluid domain $\Omega_{f}$. When considering porous media, the domain $\Omega$ is assumed fully saturated, with the fluid phase occupying the region $\Omega_{f}$ whereas the remaining part $\Omega_{s}=$ $\Omega-\Omega_{f}$ is occupied by a solid phase assumed at rest.

An appropriate constitutive equation must be postulated to describe the fluid's rheology. There are many possible choices, the most usual ones being related to Newtonian, 
generalized Newtonian and viscoelastic fluids, as summarized below.

- For a Newtonian fluid, the constitutive equation reads

$\boldsymbol{\sigma}=-p \mathbf{I}+\boldsymbol{\tau}=-p \mathbf{I}+2 \eta \mathbf{D}$,

where $p$ is the pressure field that can be interpreted as the Lagrange multiplier associated with the incompressibility constraint, $\mathbf{I}$ is the identity tensor, $\boldsymbol{\tau}$ the extra-stress tensor, $\eta$ the constant fluid viscosity and $\mathbf{D}$ the rate of strain tensor, i.e. the symmetric part of the velocity gradient, $2 \mathbf{D}=\nabla \mathbf{v}+(\nabla \mathbf{v})^{T}$.

- For a generalized Newtonian fluid, the constitutive Eq. 3 remains formally unchanged but the viscosity $\eta$ now depends on the strain rate $\dot{\gamma}$ usually expressed from the second invariant of the rate of strain tensor, i.e. $\dot{\gamma}=$ $\sqrt{2 \mathbf{D}: \mathbf{D}}$. The simplest of such models is the power-law (shear-thinning) viscosity given by

$\eta=\mathcal{K} \dot{\gamma}^{n-1}$,

where $\mathcal{K}$ and $n$ are the consistency and power-law index, respectively. The value $n=1$ corresponds to a Newtonian fluid.

- Viscoelastic models combine viscous and elastic behaviours and results in a history-dependent extrastress tensor $\boldsymbol{\tau}$. Both integral and differential models exist, but the latter are more popular in flow simulations. They have the deceptively simple generic form

$\frac{D \boldsymbol{\tau}}{D t}=\mathcal{F}(\boldsymbol{\tau}, \nabla \mathbf{v})$,

meaning that the Lagrangian or material time derivative of the extra-stress tensor is given as a model-dependent function $\mathcal{F}$ of the local extra-stress and velocity gradient evaluated along fluid trajectories.

In all these cases, suitable boundary conditions must be specified at the fluid domain boundary $\Gamma=\partial \Omega_{f}$. For inelastic fluids, they are of two types: prescribed velocity on $\Gamma_{D}$ and prescribed tractions on $\Gamma_{N}$, with $\Gamma_{D} \cup \Gamma_{N}=\Gamma$ and $\Gamma_{D} \cap \Gamma_{N}=\emptyset$. Inlet extra-stresses must also be specified for viscoelastic fluids.There is a variety of discretization techniques for solving numerically such a flow problem. The interested reader can refer to [9] and the numerous references therein. Advanced computational techniques for differential and integral viscoelastic models are detailed in [3, 12-14, 20].

Describing the internal structure of heterogeneous materials at a variety of length scales requires a high-enough resolution for capturing the size, orientation and distribution of the material constituents, i.e. the heterogeneity and its effects on the velocity field. Fibrous media with a large number of fibres and inter-fibre volumes cannot be described by considering each of these individually, which would lead to intractable boundary value problems to solve for the flow. Thus, another continuum approach at a coarser level is called for. One possibility consists in separating the scales (when this can be performed) and associating to each point $\mathbf{X} \in \Omega$ a representative volume at the finest scale $\omega(\mathbf{X})$ (with boundary $\partial \omega(\mathbf{X})$ ) from which upscaling can be carried out.

When the same physics is valid and applies at each scale, for example in the case of thermo-mechanical models, homogenization procedures work quite well in both the linear and non-linear cases. Linear and non-linear thermomechanical homogenization, including its computational counterpart, has been the subject of active research over the last three decades. The interested reader can refer to $[10,19,21]$ and the references therein. The main issue in computational homogenization is the consideration of nonlinear behaviours that involve heavy computations. Use of model order reduction techniques is an appealing route for improving the efficiency of multi-scale solvers $[4,15,16]$.

In the case of flows in porous media, the macroscopic scale corresponds to the scale of the part, much bigger than the heterogeneities scale, whereas the microscopic scale coincides with the scale of heterogeneities. In these circumstances, and for a Newtonian fluid, the macroscopic behaviour is well described by the phenomenological Darcy law that relates the fluid velocity to the pressure gradient at the macroscopic scale, whereas at the microscopic level the flow behaviour is described by the Stokes problem defined in the domain occupied by the fluid (in this work, for the sake of simplicity, we consider the solid phase as rigid and at rest). Even if both descriptions are notably different, appropriate bridges can be defined to associate to each point $\mathbf{X}$ in $\Omega$ a permeability tensor $\mathbf{K}(\mathbf{X})$ relating locally the equivalent macroscopic velocity $\mathbf{V}(\mathbf{X})$ and the pressure gradient $\nabla P(\mathbf{X})$, by upscaling the microscopic physics that only involves as material parameter the fluid viscosity $\eta$.

In [17], we derived the expressions of the macroscopic permeability in a double-scale porous medium for both Newtonian and shear-thinning fluids. In the linear case, a single microscopic calculation on a representative volume is required, which implies as many microscopic calculations as there are representative microscopic volumes in $\Omega$. In the non-linear case, and even when the microstructure can be described by a unique representative volume, the microscopic calculation must be carried out repeatedly as the microscale fluid viscosity depends on the macroscopic velocity, which in turn depends on the macroscopic permeability obtained from the microscopic calculation. In [18], we proposed an original and efficient offline-online procedure that allows one to solve the multi-scale shear-thinning fluid flow problem at a computational cost typical of a macroscopic non-linear solution. This procedure is based on the calculation of the microscopic parametric solution, 
given the velocity field at each point in the representative volume $\omega(\mathbf{X}), \mathbf{v}(\mathbf{x} \in \omega(\mathbf{X}))$, for any value of the macroscopic velocity field $\mathbf{V}, \mathbf{v}(\mathbf{x} \in \omega(\mathbf{X})$; V). From the parametric flow kinematics, parametric permeabilities $\mathbf{K}(\mathbf{V})$ were successfully and efficiently computed.

The route followed in [18] was based on the use of the so-called Proper Generalized Decomposition - PGD - widely described in our recent works [5-8]. The main idea of the PGD technique, when focusing on a given microstructure $\omega(\mathbf{X})$, consists in introducing the parameters as extra-coordinates and then solving the resulting multidimensional problem by using a separated representation of the problem solution for circumventing the curse of dimensionality that high-dimensional models entail. Within the PGD framework, the microscopic parametric velocity field reads:

$\mathbf{v}(\mathbf{x} \in \omega(\mathbf{X}), \mathbf{V}) \approx \sum_{n=1}^{Q} \mathbf{R}_{n}(\mathbf{x}) \circ \mathbf{S}_{n}(\mathbf{V})$,

where $\mathbf{R}_{n}$ and $\mathbf{S}_{n}$ are a priori unknown functions and the symbol "o" denotes the Hadamard product.

In the present paper, we extend to some of the richer rheologies encountered in industrial applications the above methodology that was successfully used in our previous works for modelling the flow of Newtonian and generalized Newtonian fluids in complex porous microstructures.

In the field of structural composites, there is a recent trend to substitute thermosets by thermoplastics. The high shear viscosity of thermoplastics, however, makes preform impregnation difficult. Two solutions are currently envisaged: use of thermoplastics with low molecular weight, and injection of a low molecular weight pre-polymer that polymerizes after having impregnated the porous medium. In both cases, the shear viscosity remains reasonably moderate to allow for preform impregnation.

The flow around fibres entails a sequence of converging and diverging flows that are likely to activate significant extensional effects. Neither the Newtonian nor the generalized Newtonian fluid models are capable of describing the behaviour of polymeric liquids in extensional flows. Consideration of richer constitutive laws seems compulsory. In the present paper, we follow the simplest route and adopt the constitutive equation of a so-called quasi-Newtonian fluid. This allows us to model realistic behaviours both in shear and extensional flows while neglecting history-dependent viscoelastic phenomena.

In Section Quasi-Newtonian fluid model, we define the quasi-Newtonian fluid model used in the present work. Then, we describe in Section Upscaling of QuasiNewtonian behaviour the upscaling procedure that allows one to go from the microscopic scale at which the quasiNewtonian model applies, to the macroscopic scale characterized by an effective Darcy law. Finally, the predicted permeabilities are discussed in Section Numerical results, and compared to those obtained with a simple generalized Newtonian fluid.

\section{Quasi-Newtonian fluid model}

The quasi-Newtonian fluid [23] differs from the generalized Newtonian fluid in the fact that the only material function involved, namely the viscosity function, is not merely a function of the second invariant of the rate of strain tensor but also depends on the relative rate of rotation of the fluid. Consequently, the quasi-Newtonian fluid is able to show shear-thinning in shear flow and extension-thickening in elongational flow. In what follows, and without loss of generality, we consider 2D planar flows.

The constitutive equation for the quasi-Newtonian fluid reads

$\boldsymbol{\sigma}=-p \mathbf{I}+2 \eta_{Q N} \mathbf{D}$,

where $\eta_{Q N}$ is the effective viscosity of the fluid that accounts for shear-thinning as well as extension-thickening according to the local type of flow.

Thus, the first step is to define a descriptor of the local flow type. There is a large variety of such descriptors [24]. In the present work, we use the particular descriptor considered in [23] and successfully validated there with the experimental results reported in [22].

First, we introduce the relative rate of rotation $\mathbf{W}$ from

$\mathbf{W}=\omega-\mathbf{w}$,

where $\omega$ is related to the flow vorticity $\boldsymbol{\Omega}(2 \boldsymbol{\Omega}=\nabla \mathbf{v}-$ $\left.(\nabla \mathbf{v})^{T}\right)$ according to

$\Omega=\epsilon \cdot \omega$,

where $\epsilon$ is the third-order permutation tensor (also known as the Levi-Civita tensor). The vector $\omega$ can also be written in terms of the curl of the velocity,

$\boldsymbol{\omega}=-\frac{1}{2} \nabla \times \mathbf{v}$.

The vector $\mathbf{w}$ in Eq. 8 represents the angular velocity of the eigenvectors of the rate of strain tensor $\mathbf{D}$. Thus, if the two eigenvectors of $\mathbf{D}$ have their orientations expressed by the unit vectors $\mathbf{d}_{1}$ and $\mathbf{d}_{2}$, then we have

$\mathbf{w}=\mathbf{d}_{i} \times \frac{D \mathbf{d}_{i}}{D t}$,

for $i=1$ or $i=2$, and where $\frac{D \bullet}{D t}$ is the material derivative.

A simple local descriptor of the type of flow can then be constructed from the second invariant of $\mathbf{D}, \dot{\gamma}=\sqrt{2 \mathbf{D}: \mathbf{D}}$, and from the norm of $\mathbf{W},\|\mathbf{W}\|$, according to

$\chi=\frac{4\|\mathbf{W}\|}{\dot{\gamma}+2\|\mathbf{W}\|}$. 
We have $0 \leq \chi \leq 2$, and more concretely

$\chi=\left\{\begin{array}{l}0, \text { in planar extension } \\ 1, \text { in pure shear } \\ 2, \text { in rigid motion }\end{array}\right.$.

Now, the shear viscosity $\eta_{S}$ depends on $\dot{\gamma}$, i.e. $\eta_{S}(\dot{\gamma})$, whereas the extensional viscosity $\eta_{E}$ depends on $\dot{\epsilon}$, with $2 \dot{\epsilon}=\dot{\gamma}$ (in 2D), i.e. $\eta_{E}(\dot{\epsilon})$. As in [23], we consider the geometrical weighting of both viscosities, affected by a function $f(\chi)$ depending on the local flow type:

$\eta_{Q N}(\dot{\gamma}, \chi)=\left(\eta_{S}(\dot{\gamma})\right)^{f(\chi)}\left(\eta_{E}(\dot{\epsilon})\right)^{1-f(\chi)}$.

Here, the function $f(\chi)$ satisfies the following conditions

$f(\chi)=\left\{\begin{array}{l}1, \text { if } \chi=1 \\ 0, \text { if } \chi=0\end{array}\right.$,

in order to recover the shear viscosity at locations exhibiting a shear flow and the extensional viscosity where planar extension occurs. We consider $f(1<\chi \leq 2)=1$ for approaching the zero shear rate viscosity in the limit case of rigid rotation.

\section{Upscaling of Quasi-Newtonian behaviour}

The upscaling procedure for Newtonian fluids, which allows one to define a bridge between the microscopic Stokes model and the macroscopic Darcy law, is summarized in the Appendix. It is adapted in [17] and [18] to handle a generalized Newtonian fluid. In what follows, we extend the procedure to the quasi-Newtonian fluid.

The quasi-Newtonian fluid flow model is solved in the representative volume $\omega(\mathbf{X})$, where two phases coexist, i.e. the fluid phase occupying the domain $\omega_{f}(\mathbf{X})$ and the solid phase, assumed rigid and at rest, occupying the region $\omega_{s}(\mathbf{X})$, with $\omega_{f}(\mathbf{X}) \cup \omega_{s}(\mathbf{X})=\omega(\mathbf{X})$ and $\omega_{f}(\mathbf{X}) \cap \omega_{s}(\mathbf{X})=$ $\emptyset$. The flow model consists of the mass and momentum balances and the quasi-Newtonian constitutive equation discussed in the previous section:

$\left\{\begin{array}{l}\nabla \cdot \boldsymbol{\sigma}=\mathbf{0} \\ \nabla \cdot \mathbf{v}=0 \\ \boldsymbol{\sigma}=-p \mathbf{I}+2 \eta_{Q N} \mathbf{D}\end{array}\right.$.

It is complemented with the boundary condition $\mathbf{v}(\mathbf{x} \in$ $\partial \omega(\mathbf{X}))=\mathbf{V}$, where the macroscopic velocity $\mathbf{V}$ is considered as an extra-coordinate in the PGD procedure. Thus, one finally obtains the solution for any possible macroscopic velocity in a certain domain $\Theta$, i.e. $\mathbf{V} \in \Theta$, where $\Theta$ is the ball (or circular disk in 2D) whose radius is set to the maximum expected velocity magnitude. It is assumed that the solid phase in $\omega(\mathbf{X})$ has an empty intersection with $\partial \omega(\mathbf{X})$, i.e. the entire boundary $\partial \omega(\mathbf{X})$ of $\omega(\mathbf{X})$ is occupied by the fluid phase. If that intersection is not empty, adequate

Fig. 1 Representative volume $\omega$

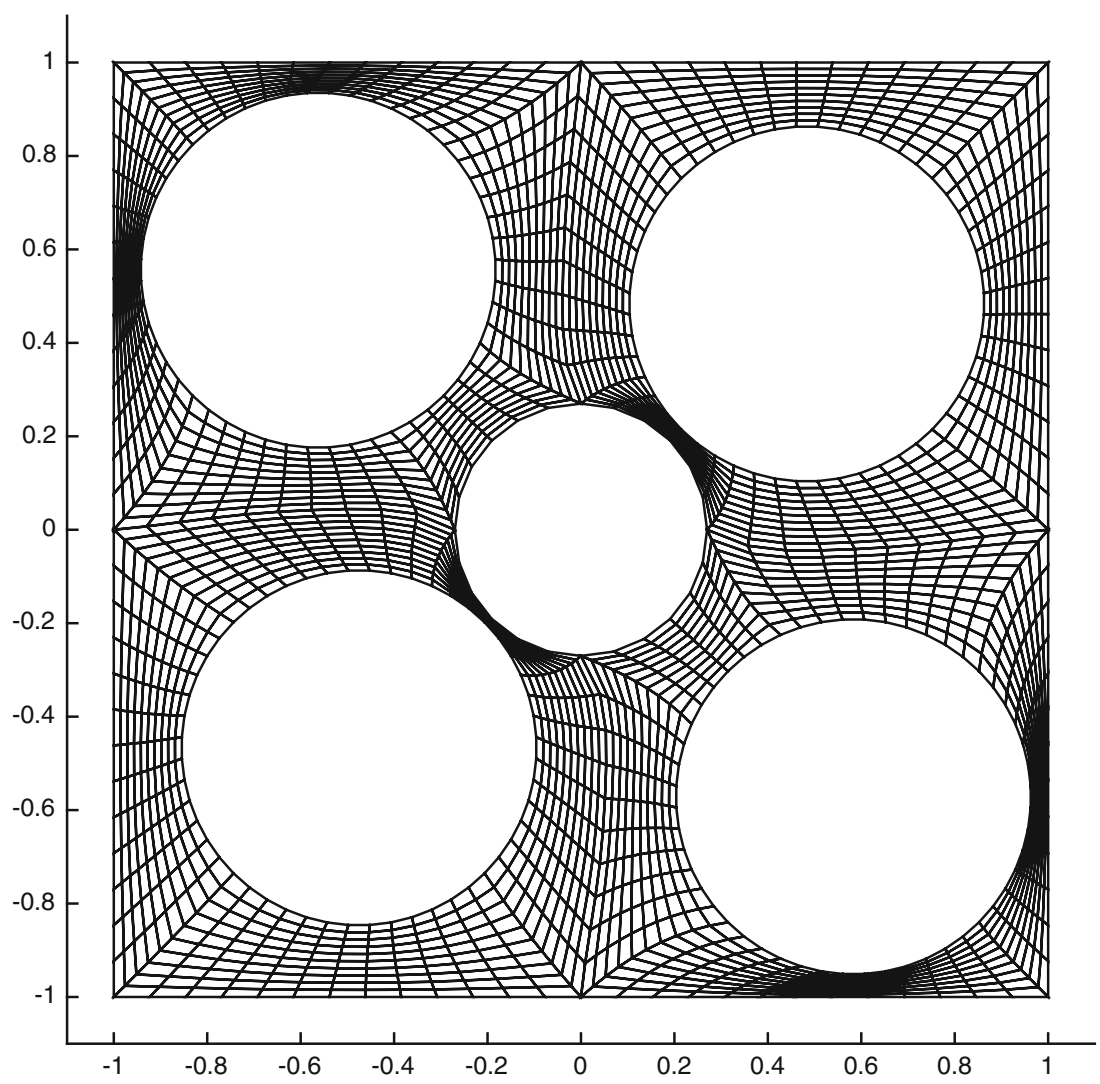


Fig. 2 Quasi-Newtonian viscosity as a function of shear and elongation rates.

Shear-thinning and extension-thickening can be noticed

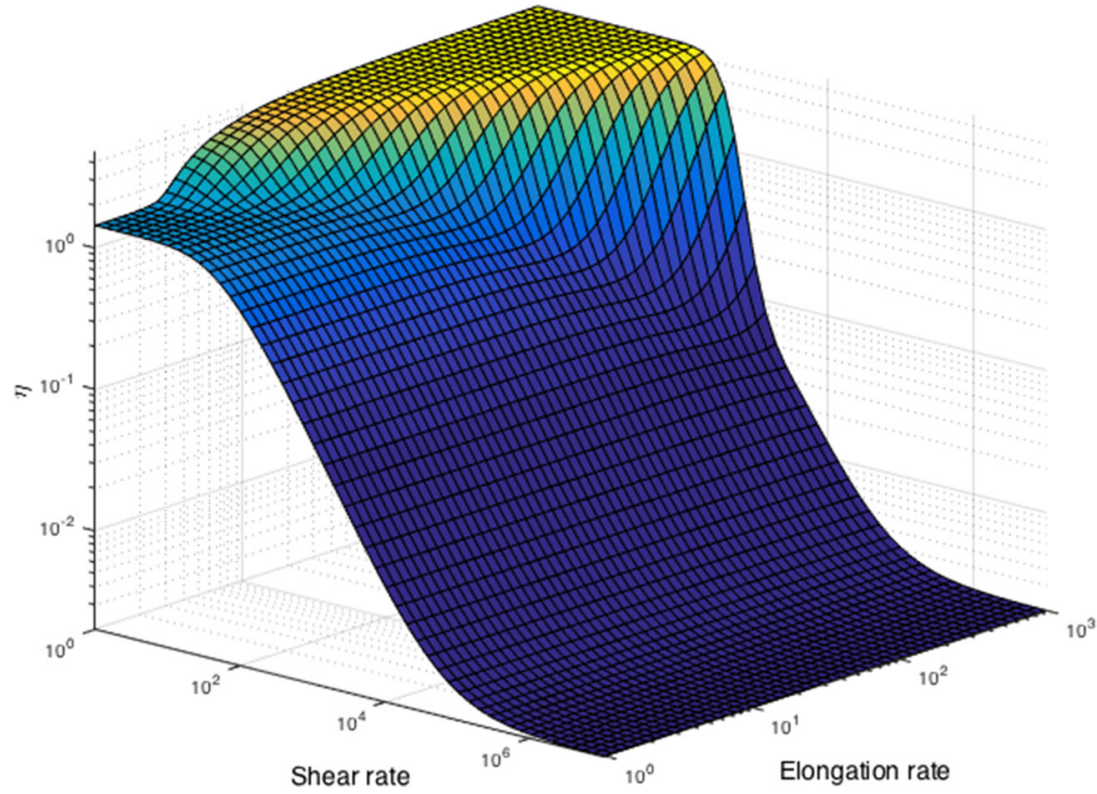

Numerical results

boundary conditions should be prescribed to ensure a vanishing velocity on $\omega_{s}(\mathbf{X}) \cup \partial \omega(\mathbf{X})$ and a flow rate equivalent to the one associated with the macroscopic velocity $\mathbf{V}$.

For a quasi-Newtonian fluid, the viscosity $\eta_{Q N}(\dot{\gamma}, \chi)$ depends on the effective strain rate $\dot{\gamma}$ and the local flow type descriptor $\chi$.

It is important to note that the flow solver considered in this work (i.e. a standard stable $Q 2 / P 1$ finite element Stokes solver for a non-uniform fluid viscosity), produces identical results when applied to the test problems considered in [23].

Once the non-linear flow problem (16) is solved for any macroscopic velocity $\mathbf{V} \in \Theta$, the parametric fluid velocity $\mathbf{v}(\mathbf{x}, \mathbf{V})$ is available. From it, $\dot{\gamma}, \dot{\epsilon}=\dot{\gamma} / 2$ and $\chi$ can be evaluated everywhere in the fluid domain $\mathbf{x} \in \omega_{f}(\mathbf{X})$ and for each macroscopic velocity $\mathbf{V} \in \Theta$ : we thus obtain $\dot{\gamma}(\mathbf{x}, \mathbf{V})$, $\dot{\epsilon}(\mathbf{x}, \mathbf{V})$ and $\chi(\mathbf{x}, \mathbf{V})$.

Then, the parametric quasi-Newtonian viscosity $\eta_{Q N}(\mathbf{x}, \mathbf{V})$ can be computed at each point in the fluid domain for each macroscopic velocity. After freezing $\eta_{Q N}(\mathbf{x}, \mathbf{V})$ at each point $\mathbf{x} \in \omega_{f}(\mathbf{X})$, the following two parametric linear Stokes problems are solved in $\omega(\mathbf{X})$ :

$\left\{\begin{array}{l}\nabla p^{i}(\mathbf{x}, \mathbf{V})=\nabla \cdot\left(2 \eta_{Q N}(\mathbf{x}, \mathbf{V}) \mathbf{D}^{i}\left(\mathbf{v}^{i}(\mathbf{x}, \mathbf{V})\right)\right) \\ \nabla \cdot \mathbf{v}^{i}(\mathbf{x}, \mathbf{V})=0\end{array}, \quad i=1,2\right.$

with the boundary conditions (in the 2D case)

$$
\left\{\begin{array}{l}
\mathbf{v}^{1}(\mathbf{x} \in \partial \omega(\mathbf{X}), \mathbf{V})=(1,0)^{T} \\
\mathbf{v}^{2}(\mathbf{x} \in \partial \omega(\mathbf{X}), \mathbf{V})=(0,1)^{T}
\end{array}\right.
$$

This yields the localization tensor, from which the parametric effective permeability $\mathbf{K}^{Q N}(\mathbf{V} ; \mathbf{X})$ can be identified, as explained in the Appendix for the case of linear fluids.
In order to apply the upscaling procedure described above, we calculate first the microscopic velocity $\mathbf{v}(\mathbf{x}, \mathbf{V})$ associated to each possible value of the macroscopic velocity $\mathbf{V}$ by means of the Proper Generalized Decomposition in order to alleviate the computational cost related to the multidimensional character of the problem. With a frozen parametric quasi-Newtonian viscosity $\eta_{Q N}(\mathbf{x}, \mathbf{V})$, the flow problems (17) become linear and their solution yields the parametric permeability $\mathbf{K}^{Q N}(\mathbf{V} ; \mathbf{X})$. For additional details on the constructor, the interested reader can refer to [18].

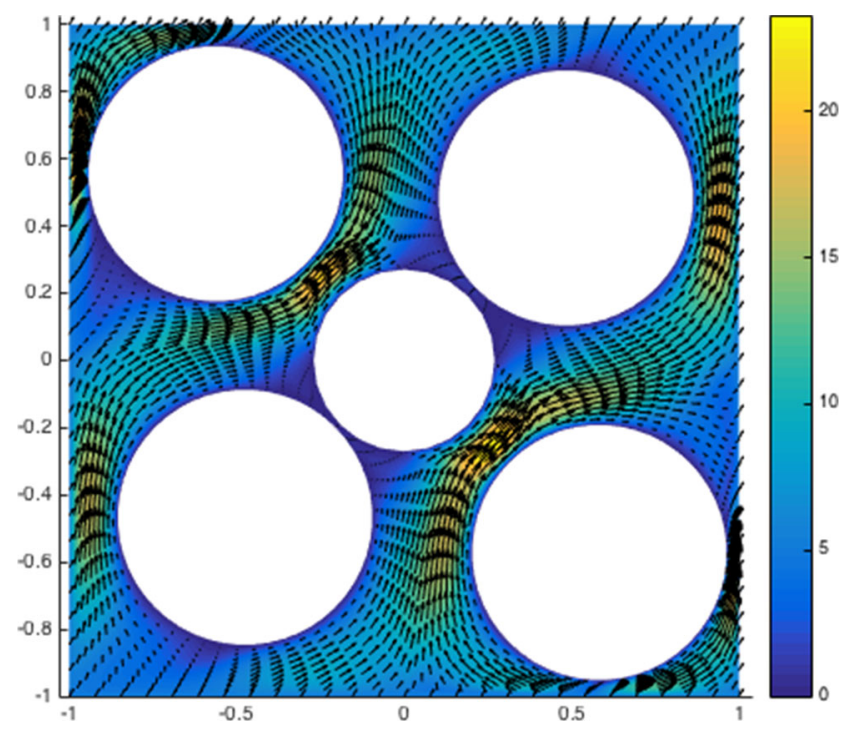

Fig. 3 Microscopic velocity field $\mathbf{v}(\mathbf{x})$ associated with the macroscopic velocity field $\mathbf{V}^{T}=(V \cos \theta, V \sin \theta), V=5 \mathrm{~mm} \cdot \mathrm{s}^{-1}$ and $\theta=\pi / 3$ 


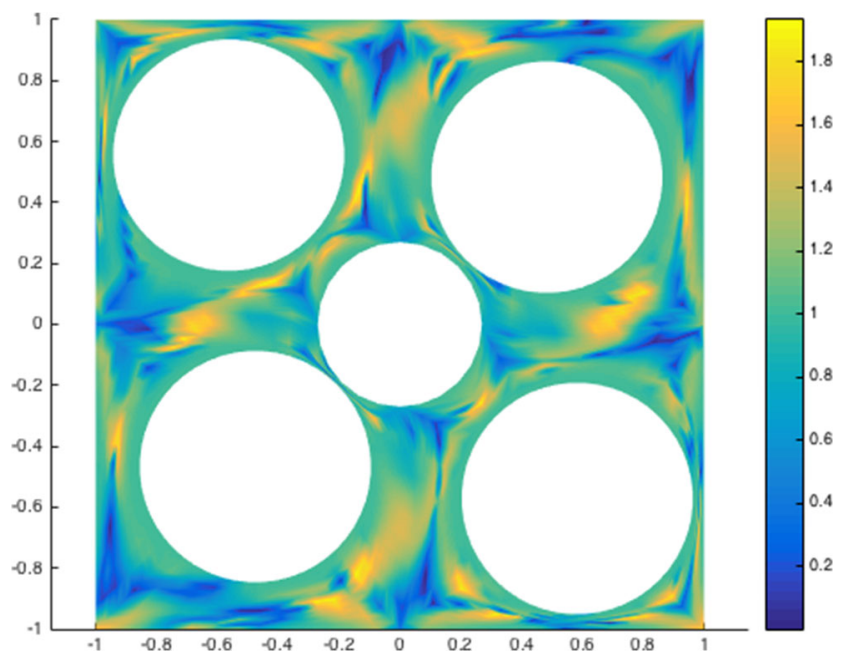

Fig. 4 Local flow descriptor $\chi(\mathbf{x})$ associated with the velocity field $\mathbf{v}(\mathbf{x})$ related to the macroscopic velocity field $\mathbf{V}^{T}=(V \cos \theta, V \sin \theta)$, $V=5 \mathrm{~mm} \cdot \mathrm{s}^{-1}$ and $\theta=\pi / 3$ : blue colour indicates elongation, yellow rigid motion and green shear

In this section, we consider the representative volume depicted in Fig. 1. It is slightly asymmetric in order to induce richer kinematics.

Following the discussion in [23], the quasi-Newtonian fluid rheology is selected such as to approximate the response of a Giesekus model with relaxation time $\lambda$, mobility $\alpha$, solvent viscosity $\eta^{s}$ and polymeric contribution $\eta^{p}$ to the zero shear rate shear viscosity. The Giesekus model has a shear-thinning shear viscosity and an extension-thickening planar elongational viscosity for $0<\alpha<0.5$. Thus, we specify

$\eta_{S}(\dot{\gamma})=\eta^{p} \beta_{S}(\dot{\gamma})+\eta^{s}$,

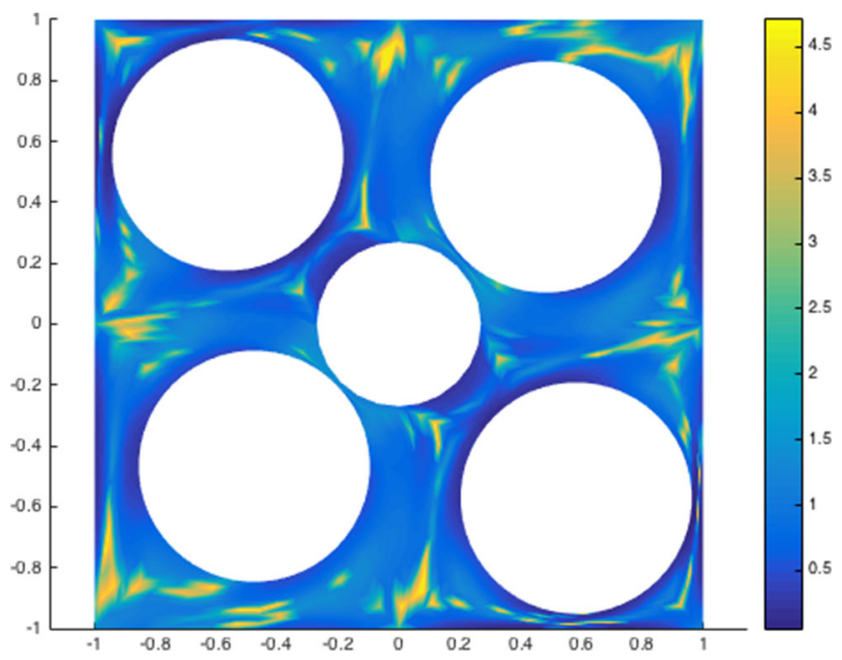

Fig. 5 Quasi-Newtonian viscosity $\eta_{Q N}$ associated with the velocity field $\mathbf{v}(\mathbf{x})$ related to the macroscopic velocity field $\mathbf{V}^{T}=$ $(V \cos \theta, V \sin \theta), V=5 \mathrm{~mm} \cdot \mathrm{s}^{-1}$ and $\theta=\pi / 3$

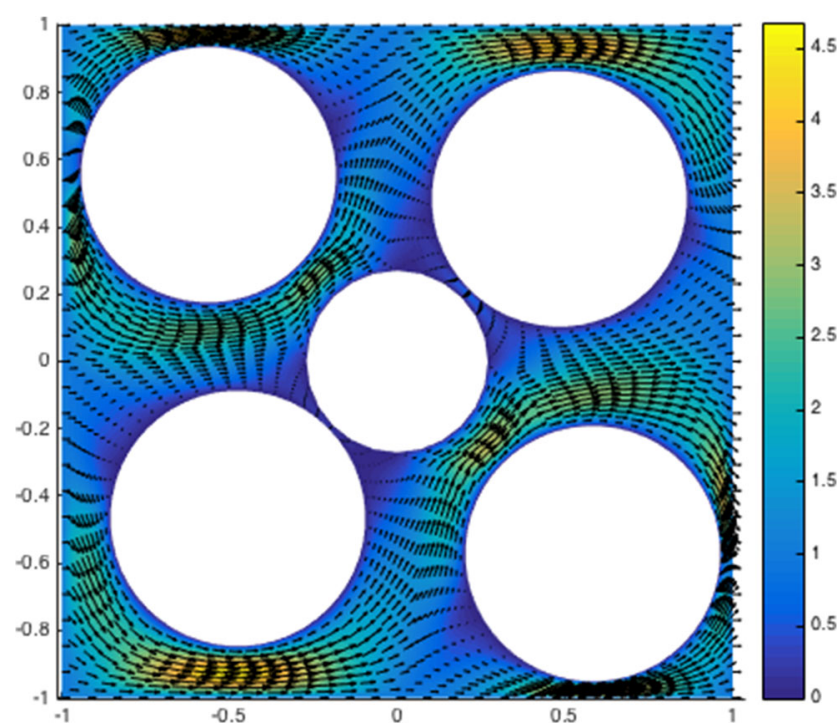

Fig. 6 Solution $\mathbf{v}^{1}(\mathbf{x}, \mathbf{V})$ of Eq. 17 associated with the quasiNewtonian viscosity related to the macroscopic velocity field $\mathbf{V}^{T}=$ $(V \cos \theta, V \sin \theta), V=5 \mathrm{~mm} \cdot \mathrm{s}^{-1}$ and $\theta=\pi / 3$

and

$\eta_{E}(\dot{\epsilon})=\eta^{p} \beta_{E}(\dot{\epsilon})+\eta^{s}$

with

$\left\{\begin{array}{l}\beta_{S}(\dot{\gamma})=\frac{y(y-1+2 \alpha)}{2 \alpha\left(y^{2}+(\lambda \dot{\gamma})^{2}\right)} \\ y^{2}=\frac{1}{2}(1+\sqrt{1+v}) \\ v=16 \alpha(1-\alpha)(\lambda \dot{\gamma})^{2}\end{array}\right.$,

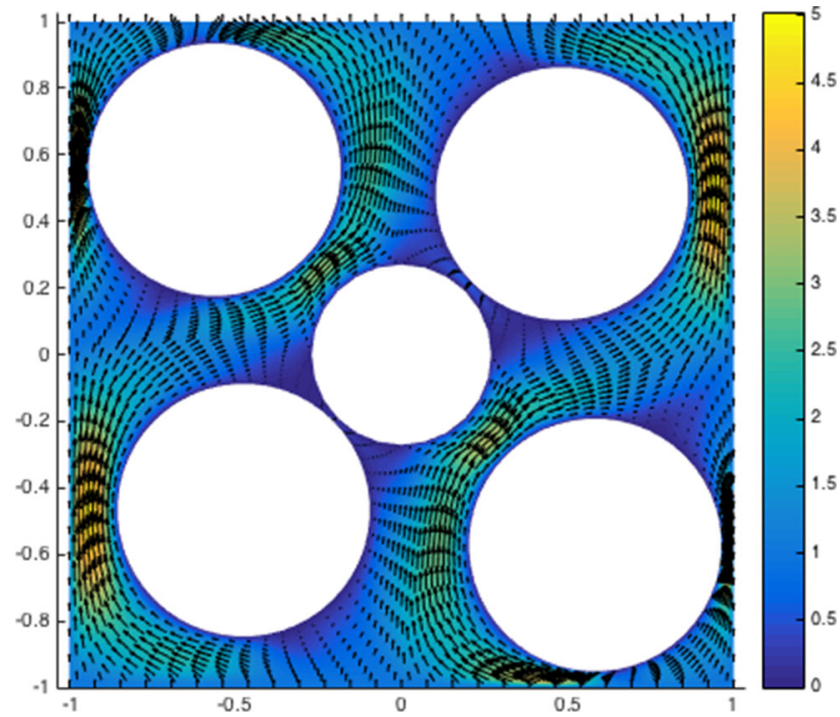

Fig. 7 Solution $\mathbf{v}^{2}(\mathbf{x}, \mathbf{V})$ of Eq. 17 associated with the quasiNewtonian viscosity related to the macroscopic velocity field $\mathbf{V}^{T}=$ $(V \cos \theta, V \sin \theta), V=5 \mathrm{~mm} \cdot \mathrm{s}^{-1}$ and $\theta=\pi / 3$ 
and

$$
\left\{\begin{array}{l}
\beta_{E}(\dot{\epsilon})=\frac{y-1+2 \alpha}{2 \alpha y} \\
y^{2}=1+\frac{1}{2}\left(\sqrt{(z+1)^{2}+v}-(z+1)\right) \\
v=64 \alpha(1-\alpha)(\lambda \dot{\epsilon})^{2} \\
z=-4(\lambda \dot{\epsilon})^{2}
\end{array} .\right.
$$

As in [23], the function $f(\chi)$ in Eq. 14 is defined as follows:

$f(\chi)=\left\{\begin{array}{ll}\frac{3 \sin ^{4}(\pi / 2) \chi}{1+2 \sin ^{4}(\pi / 2) \chi} & \text { if } \chi \leq 1 \\ 1 & \text { if } 1<\chi \leq 2\end{array}\right.$.

In the simulations, we set $\eta^{p}=1.422 \mathrm{~Pa} \cdot \mathrm{s}, \eta^{s}=$ $0.002 \mathrm{~Pa} \cdot \mathrm{s}, \alpha=0.15$ and $\lambda=0.03 \mathrm{~s}$. The resulting fluid
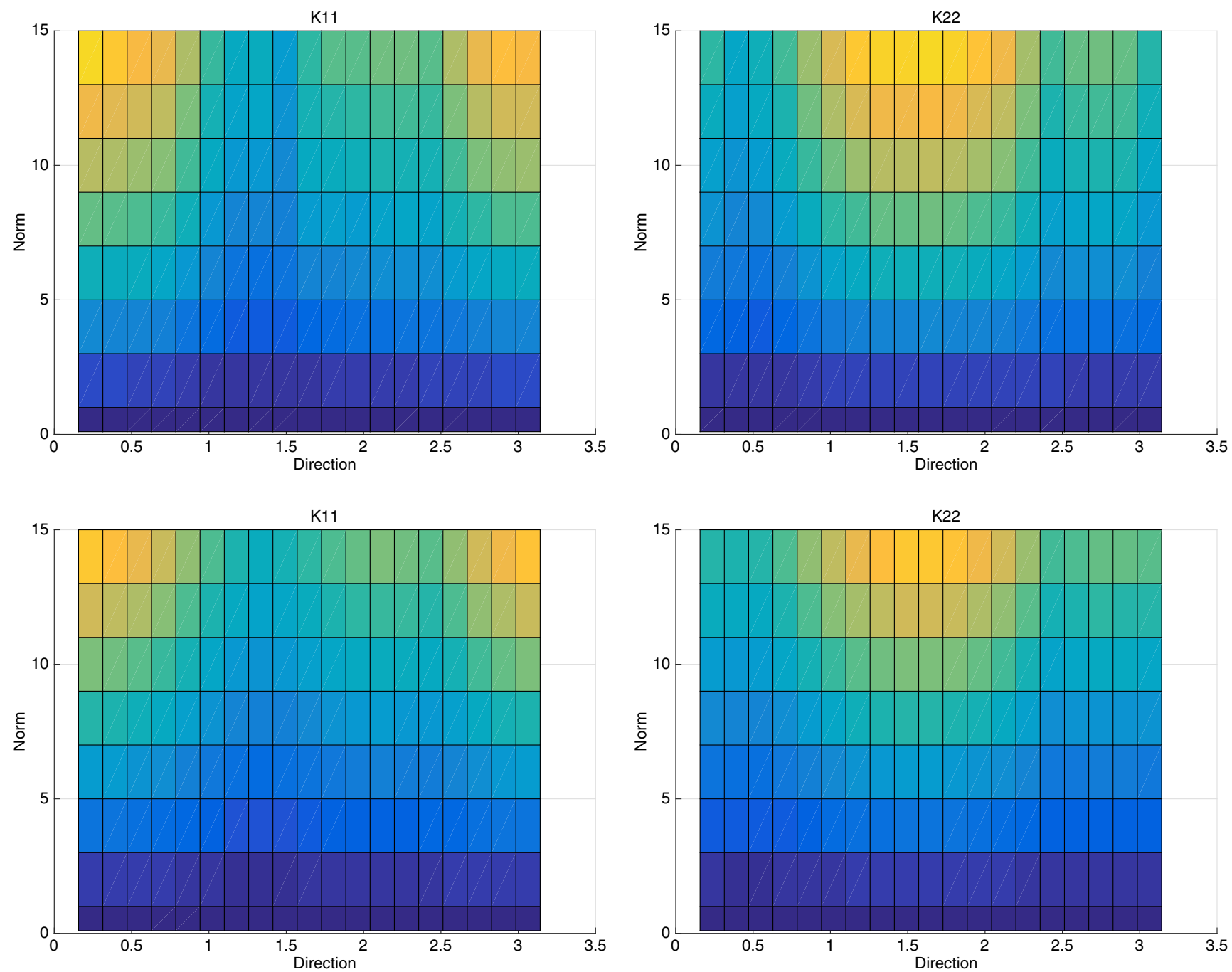

Fig. 8 Parametric effective permeability for the quasi-Newtonian (top) fluid: $\mathbf{K}_{11}^{Q N}(\mathbf{V})$ (top-left) and $\mathbf{K}_{22}^{Q N}(\mathbf{V})$ (top-right) and Generalized Newtonian fluid (bottom): $\mathbf{K}_{11}^{G N}(\mathbf{V})$ (bottom-left) and $\mathbf{K}_{22}^{G N}(\mathbf{V})$ (bottom-right) 
Fig. 9 Quasi-Newtonian viscosity as a function of shear and elongation rates for $\eta^{p}=1 \mathrm{~Pa} \cdot \mathrm{s}$ and $\eta^{s}=1 \mathrm{~Pa} \cdot \mathrm{s}$

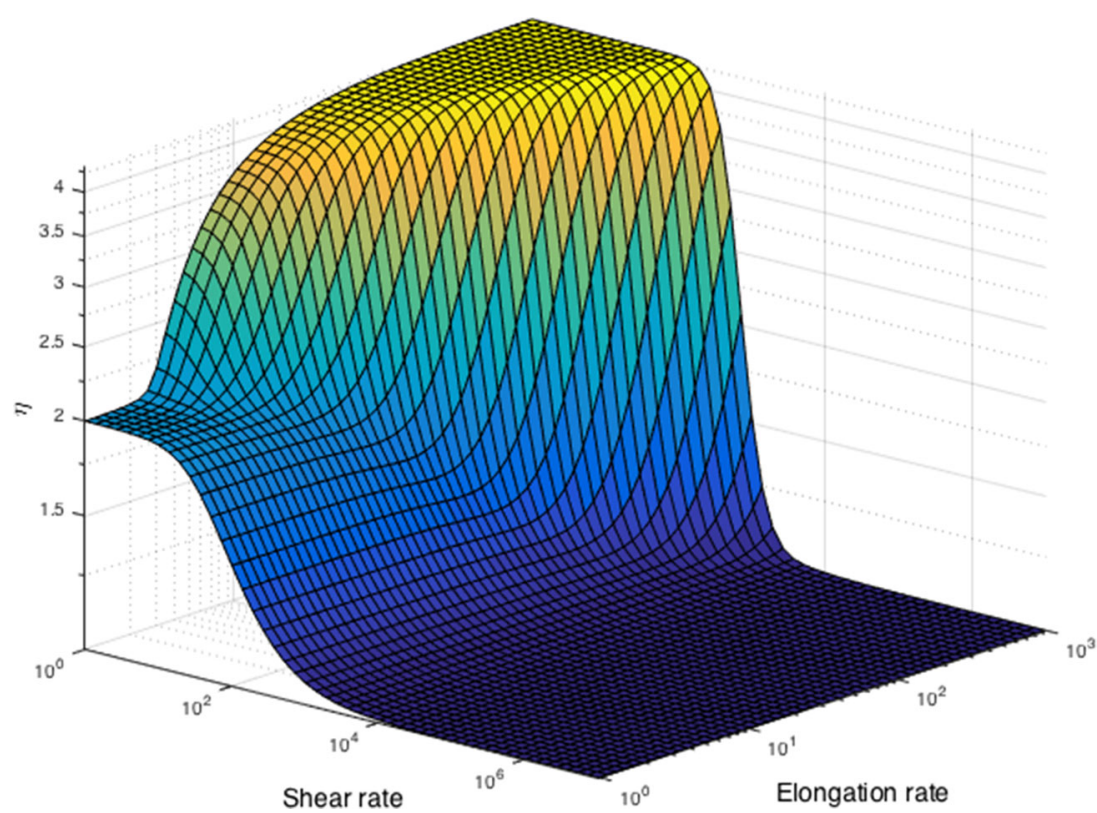

solving the two parametric linear Stokes problems (17) leading to the parametric effective permeability $\mathbf{K}^{Q N}(\mathbf{V} ; \mathbf{X})$. The solution of these two elementary flow problems associated with $\eta_{Q N}\left(\mathbf{x}, \mathbf{V}^{T}=(V \cos \theta, V \sin \theta)\right)$, with $V=$ $5 \mathrm{~mm} \cdot \mathrm{s}^{-1}$ and $\theta=\pi / 3$, are depicted in Figs. 6 and 7 .

Finally, the resulting parametric effective permeability components $\mathbf{K}_{11}^{Q N}(\mathbf{V})$ and $\mathbf{K}_{22}^{Q N}(\mathbf{V})$ are depicted in Fig. 8 (top). They are compared with the results for a Generalized Newtonian fluid with identical shear viscosity, shown in depicted in Fig. 8 (bottom) and obtained via the same procedure but by enforcing $f(\chi)=1$. We see that extensional effects have a significant influence at high flow rates, as they reduce the permeability to about half of that of the purely shear-thinning fluid. There is not, however, any noticeable effect on the principal directions of the permeability tensor.

In order to analyze the influence of the fluid rheology, we now specify $\eta^{p}=1 \mathrm{~Pa} \cdot \mathrm{s}$ and $\eta^{s}=1 \mathrm{~Pa} \cdot \mathrm{s}$, all other rheological parameters being left unchanged (Fig. 9). In view of the much larger (constant) solvent viscosity, shear-thinning is almost suppressed. Shear-thinning effects act mainly in the regions where $\chi \approx 1$, i.e. in the neighbourhood of the fluidsolid interfaces. Thus, one would expect a significant reduction of the permeability in comparison with the previous results. Moreover, a lower difference is expected between the quasi-Newtonian and Generalized-Newtonian fluids. Figure 10 compares both effective permeabilities, in particular $\mathbf{K}_{11}^{Q N}$ versus $\mathbf{K}_{11}^{G N}$, confirming the expected behaviour.
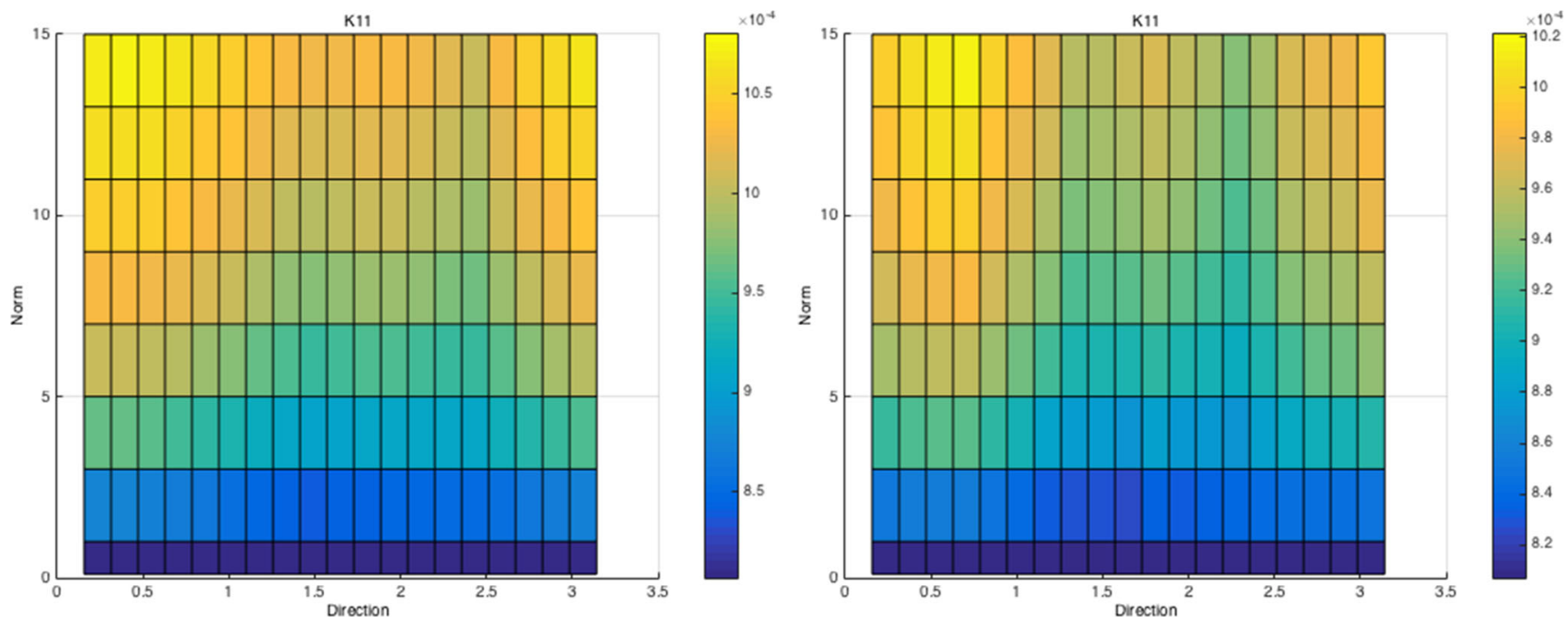

Fig. 10 Parametric effective permeability for the quasi-Newtonian and Generalized Newtonian fluids: $\mathbf{K}_{11}^{Q N}(\mathbf{V})($ left $)$ and $\mathbf{K}_{11}^{G N}(\mathbf{V})($ right $)$ 


\section{Conclusions}

In this paper, we addressed the definition of a permeability tensor for quasi-Newtonian fluids flowing in two-scale porous media. The upscaling procedure is based on the existence of a localization tensor and the equality of dissipated powers at the different scales.

For linear Newtonian fluids, an effective geometrical permeability tensor can be defined at the macroscopic scale that only depends on the microscopic geometrical features. This permeability tensor can be computed offline (for a given microstructure) by solving two boundary value problems in the 2D case. For non-linear fluids such as the Generalized Newtonian and quasi-Newtonian fluids, the permeability is no longer purely geometrical.

In order to speed up the macroscopic calculation, we proposed to construct, using the PGD, parametric solutions of microscopic flow problems defined in a representative volume with a given microstructure. Once the permeability is expressed with a parametric dependence on the macroscopic velocity, its particularization becomes computationally very fast, making it possible to solve macroscopic flow problems with a computational complexity that scales with the number of iterations needed by the non-linear macroscopic solution procedure.

Numerical results have been obtained for a quasiNewtonian model that mimics the rheological behaviour of a Giesekus viscoelastic model, showing significant shearthinning and extension-thickening. The predicted effective permeability tensor was found to be greatly affected by the extensional viscosity.

\section{Compliance with Ethical Standards}

Conflict of interests The authors declare that they have no conflict of interest.

\section{Appendix}

\section{Upscaling of linear Newtonian behaviour: from Stokes to Darcy}

Without loss of generality, suppose a statistically homogeneous porous medium where two characteristic scales coexist, the coarsest one in $\Omega$ and the finest in $\omega$, as depicted in Fig. 11. For the sake of simplicity, we assume the same microstructure everywhere in $\Omega$, which allows one to consider a unique microscopic domain $\omega$. The fluid is Newtonian and incompressible, with a constant shear viscosity $\eta$.

At the macroscopic level, the flow is described by Darcy's law and the continuity equation,

$$
\left\{\begin{array}{l}
\mathbf{V}(\mathbf{X})=-\mathbf{K}(\mathbf{X}) \cdot \nabla P(\mathbf{X}) \\
\nabla \cdot \mathbf{V}(\mathbf{X})=0
\end{array}\right.
$$

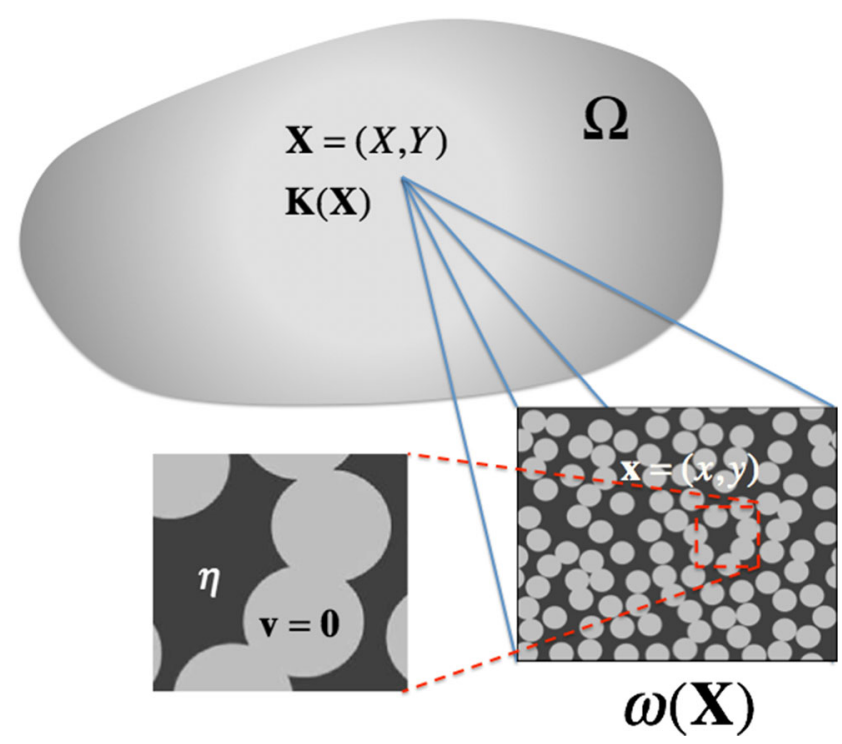

Fig. 11 Darcy (macro) and Stokes (micro) problems.

where $\mathbf{K}(\mathbf{X})$ is the macroscopic permeability tensor at position $\mathbf{X}$. In the light of the aforementioned assumption, $\mathbf{K}(\mathbf{X})$ is supposed to be the same everywhere, that is $\mathbf{K}(\mathbf{X}) \equiv \mathbf{K}$.

At the microscopic scale, inclusions occupy the solid region $\omega_{s}$ assumed rigid and at rest, i.e. $\mathbf{v}\left(\mathbf{x} \in \omega_{s}\right)=\mathbf{0}$, separated by the fluid domain $\omega_{f}$ where the Newtonian fluid flows. To be consistent with the definition of Darcy's permeability (also called intrinsic or geometric permeability), it is assumed that the fluid occupies the entire volume between solid inclusions.

In [17], the postulated macroscopic Darcy model was upscaled from the microscopic Stokes model by assuming that:

- There exists a localization tensor $\mathbf{M}(\mathbf{x}, \mathbf{X})$ such that, when the macroscopic velocity $\mathbf{V}(\mathbf{X})$ is prescribed at the boundary $\partial \omega(\mathbf{X})$ of $\omega(\mathbf{X})$, the microscopic velocity $\mathbf{v}(\mathbf{x})$ at each point $\mathbf{x} \in \omega(\mathbf{X})$ solution of the resulting Stokes problem in $\omega(\mathbf{x})$, can be obtained from

$$
\begin{aligned}
& \mathbf{v}(\mathbf{x})=\mathbf{M}(\mathbf{x}, \mathbf{X}) \cdot \mathbf{V}(\mathbf{X}), \\
& \text { with }\langle\mathbf{v}(\mathbf{x})\rangle=\frac{1}{|\omega(\mathbf{X})|} \int_{\omega(\mathbf{X})} \mathbf{v}(\mathbf{x}) d \mathbf{x}=\mathbf{V}(\mathbf{X}) .
\end{aligned}
$$

- The power dissipated at both scales must be the same for any velocity $\mathbf{V}(\mathbf{X})$. As shown in [17], this yields

$$
\langle 2 \eta \mathbf{D}: \mathbf{D}\rangle=\nabla P(\mathbf{X}) \cdot \mathbf{V}(\mathbf{X}) \text {. }
$$

In view of macroscopic Darcy's law, we thus have

$$
2 \eta\langle\mathbf{D}: \mathbf{D}\rangle=\mathbf{V}^{T}(\mathbf{X}) \cdot \mathbf{K}^{-1}(\mathbf{X}) \cdot \mathbf{V}(\mathbf{X}) .
$$

Introducing Eq. 25 in the left-hand side of Eq. 27 and developing the contraction product yields:

$$
\langle\mathbf{D}: \mathbf{D}\rangle=\left\langle D_{i j} \cdot D_{i j}\right\rangle=\frac{1}{4}\left\langle\left(v_{i, j}+v_{j, i}\right) \cdot\left(v_{i, j}+v_{j, i}\right)\right\rangle,
$$


with

$v_{i}=M_{i k} \cdot V_{k}$

with $i=1,2,3$ and $k=1,2,3$, in the general 3D case.

Thus, as soon as the localization tensor $\mathbf{M}$ is determined, from the macroscopic velocity $\mathbf{V}$ we can compute the microscopic velocity $\mathbf{v}(\mathbf{x})$ at each position $\mathbf{x}$ within the representative volume $\omega(\mathbf{X})$, and then evaluate the average of their derivatives according to Eq. 27 in order to identify $\mathbf{K}^{-1}$ and from it $\mathbf{K}$.

For calculating the localization tensor $\mathbf{M}(\mathbf{x}, \mathbf{X})$ in the general 3D case, we solve three Stokes flow problems in the microscopic domain $\omega(\mathbf{X})$ :

$\left\{\begin{array}{l}\nabla p^{i}(\mathbf{x})=\eta \nabla^{2} \mathbf{v}^{i}(\mathbf{x}) \\ \nabla \cdot \mathbf{v}^{i}(\mathbf{x})=0\end{array}\right.$,

for three different boundary conditions on $\partial \omega(\mathbf{X})$, which is considered fully-contained in the fluid phase, i.e. $\partial \omega(\mathbf{X}) \cap$ $\bar{\omega}_{s}=\emptyset$ :

$\left\{\begin{array}{l}\mathbf{v}^{1}(\mathbf{x} \in \partial \omega(\mathbf{X}))=(1,0,0)^{T} \\ \mathbf{v}^{2}(\mathbf{x} \in \partial \omega(\mathbf{X}))=(0,1,0)^{T} \\ \mathbf{v}^{3}(\mathbf{x} \in \partial \omega(\mathbf{X}))=(0,0,1)^{T}\end{array}\right.$.

These are compatible with the incompressibility constraint. Thus, the localization tensor $\mathbf{M}(\mathbf{x}, \mathbf{X})$ is finally obtain as

$\mathbf{M}(\mathbf{x}, \mathbf{X})=\left(\begin{array}{lll}\mathbf{v}^{1}(\mathbf{x}) & \mathbf{v}^{2}(\mathbf{x}) & \mathbf{v}^{3}(\mathbf{x})\end{array}\right)$.

\section{References}

1. Aghighi S, Ammar A, Metivier C, Normandin M, Chinesta F (2013) Non incremental transient solution of the Rayleigh-Bénard convection model using the PGD. J Non-Newtonian Fluid Mech 200:65-78

2. Aghighi MS, Ammar A, Metivier C, Chinesta F (2015) Parametric solution of the Rayleigh-Bénard convection model by using the PGD: Application to nanofluids. Int J Numer Methods Heat Fluid Flows 25(6): 1252-1281

3. Binetruy C, Chinesta F, Keunings R (2015) Flows in Polymers, Reinforced Polymers and Composites. A multiscale approach. Springer, Springerbriefs

4. Chinesta F, Ammar A, Lamarchand F, Beauchene P, Boust F (2008) Alleviating mesh constraints: Model reduction, parallel time integration and high resolution homogenization. Comput Methods Appl Mech Eng 197:400-413

5. Chinesta F, Ammar A, Leygue A, Keunings R (2011) An overview of the proper generalized decomposition with applications in computational rheology. J Non-Newtonian Fluid Mechanics 166:578-592

6. Chinesta F, Leygue A, Bordeu F, Aguado JV, Cueto E, Gonzalez D, Alfaro I, Ammar A, Huerta A (2013) A. PGD-based computa- tional vademecum for efficient design, optimization and control. Archives of Computational Methods in Engineering 20/1:31-59

7. Chinesta F, Keunings R, Leygue A (2013) The Proper Generalized Decomposition for Advanced Numerical Simulations. A primer. Springer, Springerbriefs

8. Chinesta F, Huerta A, Rozza G, Willcox K (2015) Model order reduction. Encyclopedia of computational mechanics. In: Stein E, de Borst R, Hughes T (eds), 2nd Edn. Wiley

9. Donea J, Huerta A (2003) Finite element methods for flow problems. Wiley

10. Geers MGD, Kouznetsova VG, Brekelmans WAM (2010) Multiscale computational homogenization: Trends and challenges. J Comput Appl Math 234/7:2175-2182

11. Ghnatios Ch, Chinesta F, Binetruy Ch (2015) 3D Modeling of squeeze flows occurring in composite laminates. Int J Mater Form 8:73-83

12. Keunings R (1989) Simulation of viscoelastic fluid flow, in Fundamentals of Computer modeling for Polymer Processing. In: Tucker CL III (ed). Carl Hanser Verlag, pp 402-470

13. Keunings R (2003) Finite Element Methods For Integral Viscoelastic Fluids. Rheology Reviews. In: Binding DM, Walters K (eds). British Society of Rheology, pp 167-195

14. Keunings R (2004) Micro-macro Methods for the Multi-Scale Simulation of Viscoelastic Flow using Molecular Models of Kinetic Theory. Rheology Reviews. In: Binding DM, Walters K (eds). British Society of Rheology, pp 67-98

15. Lamari H, Ammar A, Cartraud P, Legrain G, Jacquemin F, Chinesta F (2010) Routes for efficient computational homogenization of Non-Linear materials using the proper generalized decomposition. Archives of Computational Methods in Engineering 17/4:373-391

16. Lopez E, Abisset-Chavanne E, Lebel F, Upadhyay R, ComasCardona S, Binetruy C, Chinesta F (2016) Advanced thermal simulation of processes involving materials exhibiting fine-scale microstructures. Int J Mater Form 9:179-202

17. Lopez E, Abisset-Chavanne E, Comas-Cardona S, Binetruy et C, Chinesta F (2016) Flow modeling of linear and nonlinear fluids in two and three scale fibrous fabrics. Int J Mater Form 9:215-227

18. Lopez E, Leygue A, Abisset-Chavanne E, Comas-Cardona S, Aufrere C, Binetruy C, Chinesta F Flow modeling of linear and nonlinear fluids in two scale fibrous fabrics. Int J Mater Form. doi:10.1007/s12289-015-1280-5

19. Nguyen VP, Stroeuen M, Sluys LJ (2011) Multiscale continous and discontinous modeling of heterogeneous materials: a review on recent developments. J Multiscale Modelling 03:229

20. Owens RG, Phillips TN (2002) Computational rheology. Imperial College Press, London

21. Ponte Castañeda P, Suquet P (1998) Nonlinear composites. Adv Appl Mech 34:171-302

22. Quinzani LM, Armstrong RC, Brown RA (1994) Birefringence and laser-Doppler velocimetry (LDV) studies of viscoelastic flow through a planar contraction. J Non-Newtonian Fluid Mech 52:136

23. Ryssel E, Brunn PO (1999) Comparison of a quasi-Newtonian fluid with a viscoelastic fluid in planar contraction flow. J NonNewtonian Fluid Mech 86:309-335

24. Thompson RL, Souza Mendes PR (2005) Persistence of straining and flow classification. Int J Eng Sci 43:79-105 